\title{
Irbesartan improves endothelial dysfunction, abnormal lipid profile, proteinuria and liver dysfunction in Zucker diabetic fatty rats independent of glucose and insulin levels
}

\author{
MUNEHISA SHIMAMURA ${ }^{1}$, HIRONORI NAKAGAMI ${ }^{1}$, TAKASHI SHIMOSATO ${ }^{2}$, TOSHINORI MORITANI ${ }^{2}$, \\ FUTOSHI NAKAGAMI $^{3}$, MARIANA KIOMY OSAKO ${ }^{4}$, TAKASHI MIYAKE ${ }^{1}$, HIROSHI KORIYAMA ${ }^{3}$, \\ HIDEO SHIMIZU $^{3}$ and RYUICHI MORISHITA ${ }^{4}$ \\ ${ }^{1}$ Division of Vascular Medicine and Epigenetics, Department of Child Development, United Graduate School of Child
Development, Osaka University, Kanazawa University and Hamamatsu University School of Medicine, Kanazawa;
${ }^{2}$ Research Department, Nissei Bilis Co., Ltd., Ohtsu; Departments of ${ }^{3}$ Geriatric Medicine, and
${ }^{4}$ Clinical Gene Therapy, Graduate School of Medicine, Osaka University, Osaka, Japan
}

Received April 19, 2011; Accepted June 7, 2011

DOI: 10.3892/etm.2011.307

\begin{abstract}
Treatment with angiotensin type 1 receptor blockers (ARBs) is known to improve renal dysfunction and glucose metabolism in obese diabetic animal models and humans. This study examined the effects of irbesartan, a unique ARB with PPAR $\gamma$ activation, on endothelial dysfunction, renal dysfunction, abnormal lipid profile, and liver dysfunction in obese fa/fa Zucker diabetic fatty (ZDF) rats. ZDF rats were administered irbesartan (30 mg/kg/day p.o.) for 12 weeks. Blood pressure, glucose metabolism, lipid profile and renal function were measured every 4 weeks. Response of mesenteric artery rings to acetylcholine was also evaluated as an index of endothelial function after 12 weeks of treatment. Although irbesartan did not affect glucose and insulin levels in both glucose and insulin tolerance tests, decreases in systolic blood pressure, dyslipidemia, and urinary protein excretion were noted from 4 weeks after the start of treatment and continued until 12 weeks. Endothelial and liver dysfunctions were also improved after 12 weeks of treatment. Compared to previous reports showing the effects of irbesartan at later time points such as 6 or 12 months, the present study demonstrated that a low-dose of irbesartan had favorable effects from the early period of treatment, independent of glucose metabolism. Our findings suggest that a low-dose of irbesartan improves diabetic complications quickly after starting treatment, and may support the use of irbesartan for preventing progression of diabetic complications.
\end{abstract}

Correspondence to: Professor Ryuichi Morishita, Department of Clinical Gene Therapy, Graduate School of Medicine, Osaka University, 2-2 Yamada-oka, Suita, Osaka 565-0871, Japan

E-mail: morishit@cgt.med.osaka-u.ac.jp

Key words: type 2 diabetes, renal dysfunction, endothelial dysfunction, dyslipidemia, AT1 receptor blocker

\section{Introduction}

Diabetic angiopathy, nephropathy, and dyslipidemia are important risk factors for multiple organ dysfunctions such as ischemic heart disease and chronic renal failure. There is growing evidence that angiotensin (Ang) II plays a key role in the activation of inflammation and oxidative stress, which accelerate the progression of such diseases through the Ang II type 1 receptor (AT1R) (1-3). Based on this viewpoint, the prevention of Ang II signaling by AT1R blockers (ARBs) has been extensively used in diabetic patients $(1,2)$. Among them, irbesartan, a so-called metabosartan, is a unique ARB with the ability to increase the transcriptional activity of PPAR- $\gamma$, which was found to decrease pro-inflammatory cytokines in monocytes (4) and prevent up-regulation of MCP-1 receptor expression in animal studies (5). Since irbesartan stimulated PPAR- $\gamma$ activity in an AT1R-deficient cell model, the stimulatory effect of irbesartan on PPAR- $\gamma$ activity is independent of its AT1R blockade (6). Importantly, two large prospective, randomized, double-blind clinical trials demonstrated that irbesartan prevented the onset (7) and progression of chronic kidney disease (8), independent of its blood pressure-lowering effect. Additionally, endothelial dysfunction was significantly improved by short-term atorvastatin and/or irbesartan in type 2 diabetic patients without affecting blood pressure (2). These data suggest that irbesartan could have organ-protective actions independent of blood pressure lowering, possibly due to PPAR- $\gamma$ activation, since PPAR- $\gamma$ agonists such as pioglitazone demonstrated prevention of cardiovascular events in several clinical trials (9). To investigate the potential beneficial effects of PPAR- $\gamma$ activation by irbesartan, we examined whether diabetic complications are improved by irbesartan in ZDF rats.

\section{Materials and methods}

Animals. All procedures were approved by the Institutional Animal Care and Use Committee of Osaka University. Exper- 
Table I. Body weight, blood pressure, food intake and glucose level.

\begin{tabular}{|c|c|c|c|c|c|c|c|c|c|}
\hline & \multicolumn{3}{|c|}{ Baseline } & \multicolumn{3}{|c|}{4 weeks } & \multicolumn{3}{|c|}{12 weeks } \\
\hline & Lean & $\mathrm{ZDF}$ & $\mathrm{ZDF}+\mathrm{IRB}$ & Lean & $\mathrm{ZDF}$ & ZDF+IRB & Lean & $\mathrm{ZDF}$ & $\mathrm{ZDF}+\mathrm{IRB}$ \\
\hline Body weight & $302.8 \pm 1.8$ & $382.5 \pm 9^{\mathrm{a}}$ & $381 \pm 9.3^{\mathrm{a}}$ & $363.5 \pm 1.8$ & $393.4 \pm 10^{\mathrm{a}}$ & $392.3 \pm 10.4^{\mathrm{a}}$ & $400.8 \pm 3.4$ & $403.7 \pm 10.4$ & $416.7 \pm 7.3$ \\
\hline $\mathrm{SBP}(\mathrm{mmHg})$ & $139 \pm 2$ & $133 \pm 4$ & $129 \pm 4$ & $136 \pm 2$ & $138 \pm 2$ & $129 \pm 2^{b}$ & $133 \pm 3$ & $135 \pm 4$ & $122 \pm 2^{\mathrm{b}}$ \\
\hline Food intake (g/d) & $16.5 \pm 1.1$ & $35.6 \pm 2.4^{\mathrm{a}}$ & $33.3 \pm 1.1^{\mathrm{a}}$ & $16.9 \pm 0.6$ & $31.4 \pm 1.1^{\mathrm{a}}$ & $33.3 \pm 1.5^{\mathrm{a}}$ & $17.0 \pm 0.6$ & $37.6 \pm 2.7^{\mathrm{a}}$ & $38.0 \pm 2.1^{\mathrm{a}}$ \\
\hline Glucose (mg/dl) & $142 \pm 11$ & $266 \pm 26^{\mathrm{a}}$ & $271 \pm 21^{\mathrm{a}}$ & $139 \pm 5$ & $342 \pm 43^{\mathrm{a}}$ & $369 \pm 31^{\mathrm{a}}$ & $188 \pm 11$ & $491 \pm 24^{\mathrm{a}}$ & $512 \pm 39^{\mathrm{a}}$ \\
\hline
\end{tabular}

IRB, irbesartan; ZDF, Zucker diabetic fatty rat; SBP, systolic blood pressure. Values are mean \pm SEM. ${ }^{a} \mathrm{P}<0.05$ vs. lean rats, ${ }^{b} \mathrm{P}<0.05 \mathrm{vs}$. ZDF rats $(n=6$ in each case).

Table II. Oral glucose tolerance test (OGTT) and insulin tolerance test (ITT) after 10 weeks of treatment.

\begin{tabular}{|c|c|c|c|c|c|c|c|}
\hline & \multicolumn{4}{|c|}{ OGTT glucose (mg/dl) } & \multicolumn{3}{|c|}{ ITT glucose $\%$ vs. 0 min } \\
\hline & $0 \mathrm{~min}$ & $30 \mathrm{~min}$ & $60 \mathrm{~min}$ & $120 \mathrm{~min}$ & $15 \mathrm{~min}$ & $30 \mathrm{~min}$ & $60 \mathrm{~min}$ \\
\hline Lean & $107 \pm 5$ & $213 \pm 7$ & $227 \pm 6$ & $145 \pm 6$ & $42 \pm 6$ & $33 \pm 3$ & $52 \pm 4$ \\
\hline $\mathrm{ZDF}$ & $368 \pm 44^{\mathrm{a}}$ & $656 \pm 56^{a}$ & $653 \pm 25^{\mathrm{a}}$ & $535 \pm 25^{a}$ & $80 \pm 2^{\mathrm{a}}$ & $44 \pm 3^{a}$ & $37 \pm 3^{\mathrm{a}}$ \\
\hline ZDF+IRB & $380 \pm 33^{\mathrm{a}}$ & $699 \pm 22^{\mathrm{a}}$ & $647 \pm 23^{\mathrm{a}}$ & $539 \pm 15^{a}$ & $77 \pm 2^{\mathrm{a}}$ & $50 \pm 4^{\mathrm{a}}$ & $39 \pm 4^{\mathrm{a}}$ \\
\hline
\end{tabular}

IRB, irbesartan; ZDF, Zucker diabetic fatty rat; SBP, systolic blood pressure. Values are mean \pm SEM. ${ }^{\text {a }}<0.05$ vs. lean rats. ( $\mathrm{n}=6$ in each case).
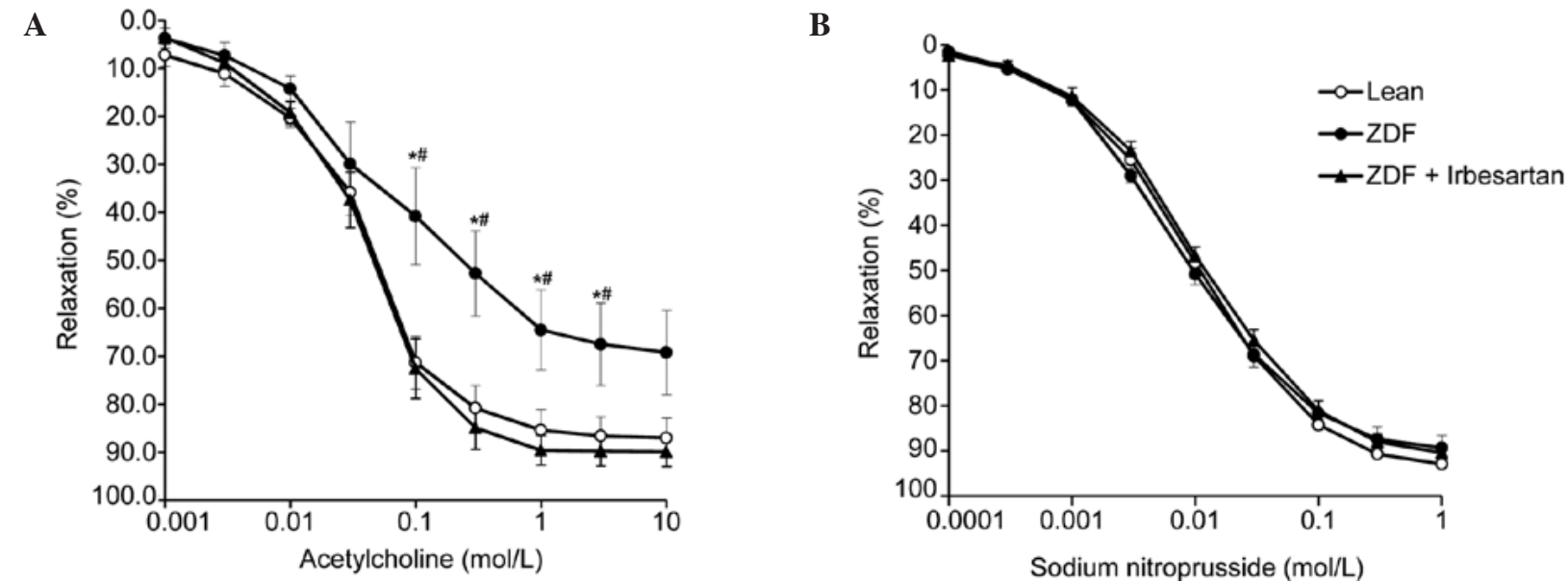

Figure 1. Cumulative concentration-response curves to acetylcholine (A) and sodium nitroprusside (B) for isolated mesenteric artery rings pre-contracted with $3 \times 10^{-6} \mathrm{~mol} / 1$ phenylephrine. Relaxation rates were calculated as the percentages of maximum relaxation induced by $10^{-4}$ mol/1 papaverine. Points and vertical bars represents the mean \pm SE of 6 preparations. ${ }^{*} \mathrm{P}<0.05$ vs. untreated-ZDF rats; ${ }^{\#} \mathrm{P}<0.05$ vs. lean rats.

iments were performed in obese ZDF rats (12 weeks old) from Charles River Japan (Yokohama, Japan). As the control, lean $\mathrm{fa} /+$ Zucker rats were used. Irbesartan was purchased from Shionogi Co., Ltd. (Osaka, Japan). The animals were divided into three groups: lean rats, ZDF rats and ZDF rats administered $30 \mathrm{mg} / \mathrm{kg} /$ day of irbesartan by gavage for 12 weeks. Systolic blood pressure was measured at $0,4,8$ and 12 weeks by the tail-cuff method (MK-2000; Muromachi Kikai, Ltd., Tokyo, Japan). Fasting blood samples and 24-h urine samples were collected at $0,4,8$ and 12 weeks. Serum total cholesterol, triglyceride, BUN, creatinine, AST, ALT and urinary protein excretion were measured using commercially available kits (Wako Pure Chemical, Osaka, Japan).

Measurement of food intake and water consumption. For food and water intake measurement, one or two rats were housed in a cage. A preweighed amount of food was provided, and the weight consumed (evaluated as the difference between the original amount and the food left in the cage, including spillage) was measured carefully every $24 \mathrm{~h}$ for 5 days. Similarly, water intake was determined as the difference in weight of water in the bottle every $24 \mathrm{~h}$ for 5 days. 


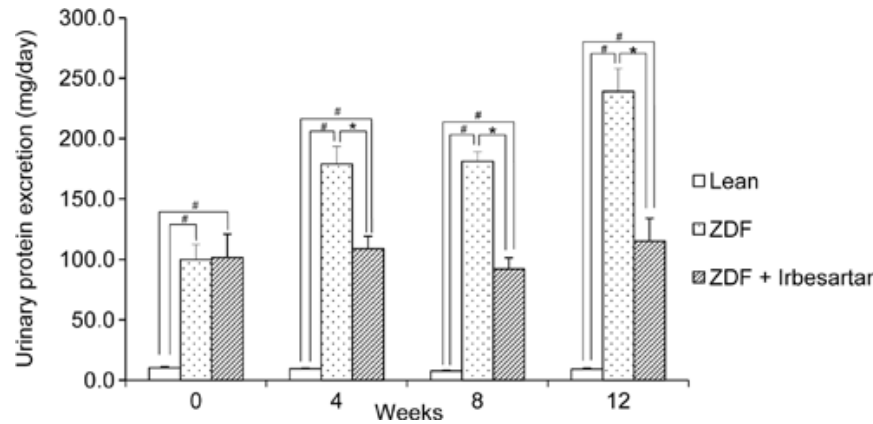

Figure 2. Effects of irbesartan on urinary protein excretion during 12 weeks follow-up. ${ }^{\mathrm{P}}<0.05$ vs. untreated-ZDF rats; ${ }^{~} \mathrm{P}<0.05$ vs. lean rats; ${ }^{\#} \mathrm{P}<0.05$ vs. lean rats.

The blood glucose level $(\mathrm{mg} / \mathrm{dl})$ was measured by the glucose oxidase method using an Antosense (Horiba, Ltd., Kyoto, Japan). For the glucose tolerance test (GTT), rats were fasted overnight and injected orally with glucose $(2 \mathrm{~g} / \mathrm{kg})$. For the insulin tolerance test (ITT), rats in the fasted state were injected intravenously with $0.75 \mathrm{U} / \mathrm{kg}$ human regular insulin (Novolin R; Novo Nordisk). Blood samples were collected from the tail vein before and at different time points after injection as indicated in the figures.

Preparation of mesenteric artery rings and evaluation of endothelial function. Rats were sacrificed at 12 weeks, and mesenteric artery rings were dissected and fixed vertically between hooks in 10-ml organ baths containing Krebs-Henseleit buffer $\left(118 \mathrm{mM} \mathrm{NaCl}, 4.7 \mathrm{mM} \mathrm{KCl}, 2.5 \mathrm{mM} \mathrm{CaCl}_{2}, 1.2 \mathrm{mM}\right.$

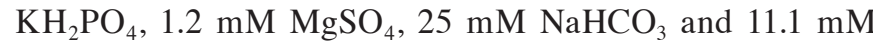
glucose) aerated with a mixture of $95 \% \mathrm{O}_{2}$ and $5 \% \mathrm{CO}_{2}$ and maintained at $37^{\circ} \mathrm{C}$. To prevent the synthesis of vascular prostaglandins, the buffer contained indomethacin $\left(10^{-5} \mathrm{M}\right)$. The hook anchoring the upper end of the strips was connected to an isometric transducer (TB-611T; Nihon-Kohden, Tokyo, Japan). The resting tension was adjusted to $1 \mathrm{~g}$. The preparations were equilibrated for at least $60 \mathrm{~min}$. During this period, the buffer was replaced every $10 \mathrm{~min}$. Concentration-response curves for acetylcholine (ACh; $10^{-9}$ to $10^{-5} \mathrm{M}$ ) were obtained in preparations partially contracted with L-phenylephrine $\left(3 \times 10^{-7}\right.$ to $3 \times 10^{-6} \mathrm{M}$ ). The relaxant response to sodium nitroprusside (SNP; $10^{-10}$ to $10^{-6} \mathrm{M}$ ) was also obtained after $10 \mathrm{~min}$ of pretreatment with L-NAME $\left(10^{-4} \mathrm{M}\right)$. Ach- or SNP-induced relaxation was expressed as a percentage of the papaverine $\left(10^{-4} \mathrm{M}\right)$-induced maximum relaxation.

Statistical analysis. All values are expressed as mean \pm SEM. One-way analysis of variance (ANOVA), followed by Tukey's multiple comparison test was used to analyze differences. In all cases, $\mathrm{P}<0.05$ was considered statistically significant.

\section{Results}

ZDF rats showed a significant increase in serum glucose level and food intake during 12 weeks (Table I), while there was no difference between the control and irbesartan groups. Similarly, water intake and body weight did not show a significant difference in both the latter groups (data not shown). In addition, there was no significant difference in ITT and GTT results at 10 weeks between the latter groups (Table II). However, treatment with irbesartan significantly improved the impaired endothelium-dependent relaxation response of isolated mesenteric artery rings, while the relaxation response to acetylcholine was significantly impaired in the ZDF rats (Fig. 1A). In contrast, the response to sodium nitroprusside was the same in all groups (Fig. 1B). Systolic blood pressure was not different between ZDF rats and lean rats, whereas rats treated with irbesartan showed lower systolic blood pressure (Table I).

During 12 weeks, urinary protein excretion was also examined. ZDF rats showed higher excretion than that of the lean rats (Fig. 2). However, ZDF rats treated with irbesartan showed no progression of urinary protein excretion (Fig. 2). Serum BUN and creatinine were not different between the treated and untreated rats (data not shown). Then, we examined whether irbesartan affected abnormal lipid profile. Serum total cholesterol and triglyceride gradually worsened until 8 weeks in ZDF rats. A significantly lower triglyceride level was observed in the irbesartan-treated rats at 12 weeks (Fig. 3). Similarly,
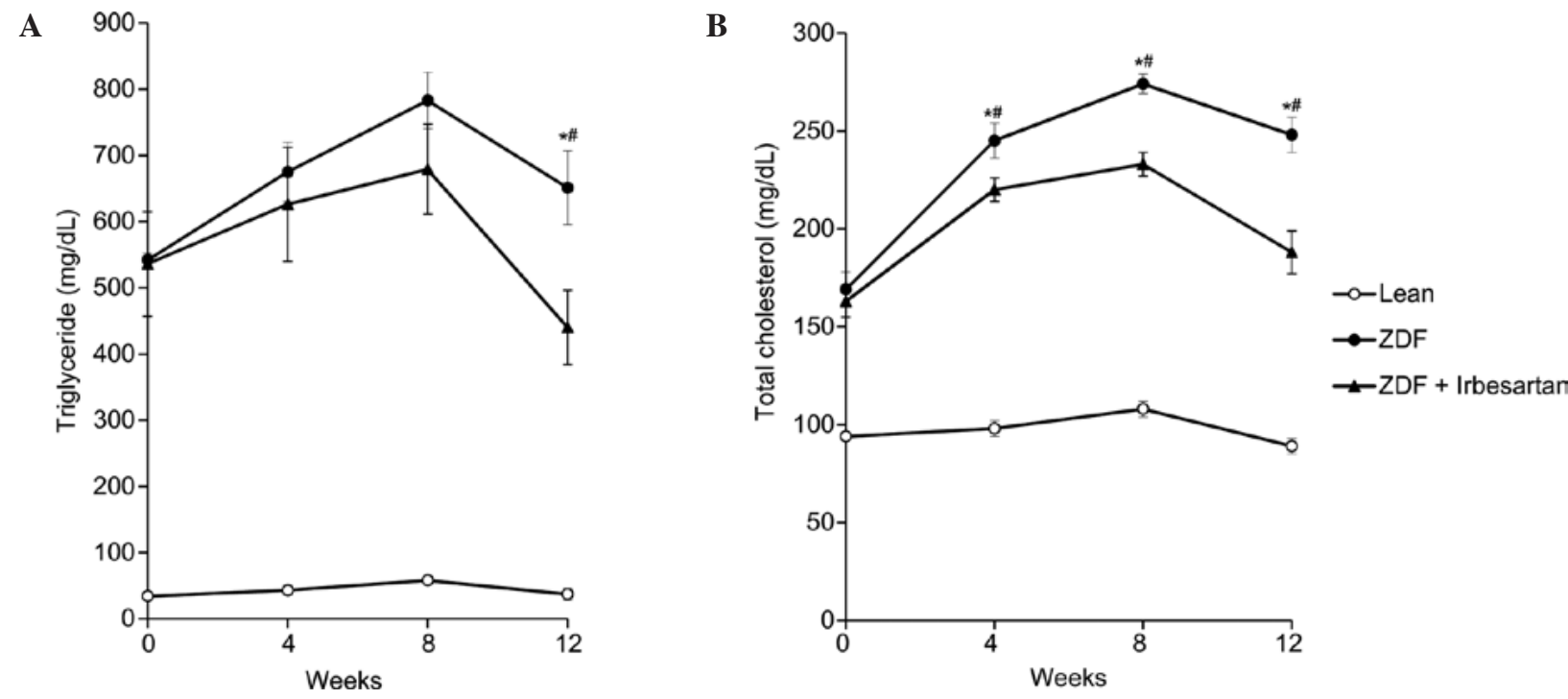

Figure 3. Effects of irbesartan on lipid profiles. (A) Serum triglyceride, (B) serum total-cholesterol. ${ }^{*} \mathrm{P}<0.05$ vs. untreated-ZDF rats; ${ }^{\text {} P}<0.05$ vs. lean rats. 


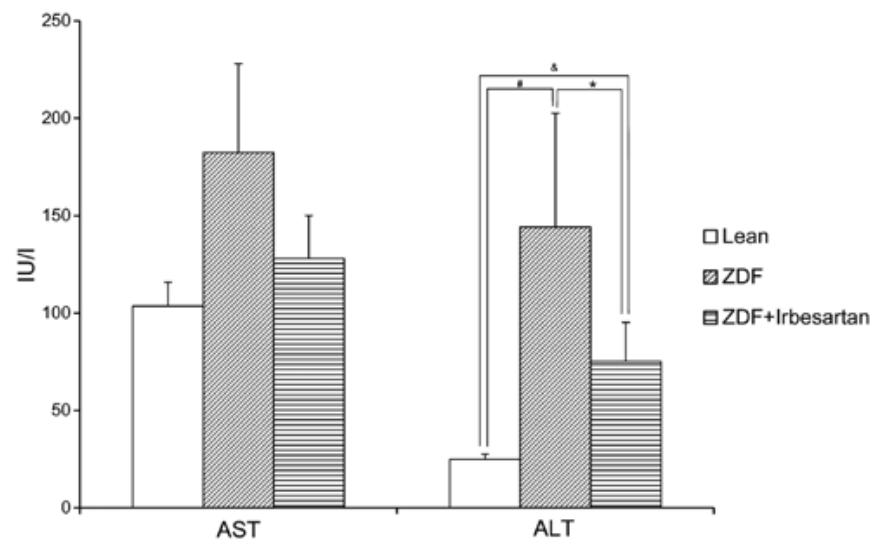

Figure 4. Effects of irbesartan on liver dysfunction at 12 weeks after treatments. ${ }^{*} \mathrm{P}<0.05$ vs. untreated-ZDF rats; ${ }^{*} \mathrm{P}<0.05$ vs. lean rats; ${ }^{\circledR} \mathrm{P}<0.05$ vs. lean rats.

the total cholesterol level was also reduced from 4 weeks after treatment (Fig. 3). Of importance, ZDF rats showed a significant increase in ALT (Fig. 4). However, the level of ALT was significantly decreased by irbesartan (Fig. 4).

\section{Discussion}

The present study demonstrated that endothelial dysfunction, proteinuria, abnormal lipid profile and liver dysfunction were significantly improved by irbesartan in ZDF rats, independent of plasma glucose and insulin levels. Endothelial dysfunction is caused by diabetes, and also accelerates the progression of diabetes. This means that the vessel endothelium is an important target tissue in diabetic patients. Thus, improvement of endothelial dysfunction is believed to be beneficial for reducing cardiovascular events in diabetic patients. In this study, administration of irbesartan for 3 months significantly attenuated endothelial dysfunction, with no effect on glucose metabolism. This finding suggests that irbesartan, as a metabosartan that activates PPAR $\gamma$, may improve endothelial dysfunction in human hypertensive patients, even if it does not lower blood glucose or insulin level. In contrast, previous reports showed that irbesartan improved plasma glucose level and insulin resistance (10-12) and decreased body weight (10). The reason for the lack of effects on glucose metabolism and serum renal parameters was probably that the dose of irbesartan in the present study was lower than that in previous reports $(50 \mathrm{mg} / \mathrm{kg})$, and the treatment period was shorter than in previous reports (12). Our study also showed that urinary protein was reduced by irbesartan, although serum renal markers and plasma glucose and insulin were not changed. Similarly, long-term administration of irbesartan in rats was reported to diminish the elevation in urinary protein excretion, plasma creatinine and urea nitrogen levels and reduce the extent of glomerular and tubule-interstitial lesions (10).

Another important finding of the present study is that treatment with irbesartan improved the abnormal lipid profile and liver dysfunction caused by non-alcoholic steatohepatitis (NASH) in ZDF rats. Since NASH is frequently present in patients with type 2 diabetes mellitus and leads to liver-related morbidity and mortality (13), the quick recovery of steatohepatitis by irbesartan may also be beneficial in diabetic patients.
Longer-term and higher-dose irbesartan $(50 \mathrm{mg} / \mathrm{kg})$ was reported to improve fat deposits through recovery of the insulin signaling pathways in the liver (14). However, the present study demonstrated that even lower-dose and shorter treatment with irbesartan may provide beneficial effects on liver dysfunction. Although irbesartan slightly lowered blood pressure in $\mathrm{ZDF}$ rats, these favorable outcomes may not have been due to blood pressure lowering, but to improvement of abnormal lipid profile by irbesartan through activation of PPAR- $\gamma$. As a PPAR- $\gamma$ agonist has been reported to decrease pro-inflammatory cytokines in monocytes (4) and prevent up-regulation of MCP-1 receptor expression in lesional and circulating monocytes (5), the decrease in pro-inflammatory gene expression through activation of PPAR- $\gamma$ by irbesartan might reduce inflammation in the endothelium, kidney and liver.

In clinical practice, the effects of irbesartan to inhibit the onset and progression of diabetic nephropathy were demonstrated in two prospective, randomized, double-blind clinical trials, without a reduction in blood pressure $(7,8)$. Sub-analysis showed that irbesartan treatment exhibited a significant decrease in hs-CRP, IL- 6 and albumin excretion, indicating that it reduces the risk of microvascular and macrovascular disease through a reduction of inflammation (1). Also, Ceriello et al (2) reported that the decrease in endothelial dysfunction, as assessed by flow-mediated vasodilation (FMD), while the increase in nitrotyrosine, C-reactive protein, intercellular adhesion molecule-1 and interleukin-6, were significantly attenuated by short-term atorvastatin and/or irbesartan treatment in type 2 diabetic patients without affecting blood pressure. From these viewpoints, the pleiotropic effects of irbesartan through PPAR- $\gamma$ activation may contribute to the better outcome, even in clinical situations.

In summary, the present study demonstrated that irbesartan significantly improved endothelial dysfunction, abnormal urinary excretion, abnormal lipid profile, and liver dysfunction in ZDF rats, without changes in glucose and insulin levels. These beneficial effects of irbesartan on diabetic nephropathy and NASH, in addition to its potent blood pressure-lowering effect, would provide a much better outcome of reduced cardiovascular events in diabetic and metabolic disease patients.

\section{Acknowledgements}

This study was partially supported by the Ministry of Education, Culture, Sports, Science and Technology, the Takeda Science Foundation, the Mitsubishi Research Foundation, and a Japan Heart Foundation/Novartis Grant for Research Award in Molecular and Cellular Cardiology, 2011.

\section{References}

1. Persson F, Rossing P, Hovind P, et al: Irbesartan treatment reduces biomarkers of inflammatory activity in patients with type 2 diabetes and microalbuminuria: an IRMA 2 substudy. Diabetes 55: 3550-3555, 2006

2. Ceriello A, Assaloni R, Da Ros R, et al: Effect of atorvastatin and irbesartan, alone and in combination, on postprandial endothelial dysfunction, oxidative stress, and inflammation in type 2 diabetic patients. Circulation 111: 2518-2524, 2005.

3. Fliser D, Buchholz K and Haller H: Antiinflammatory effects of angiotensin II subtype 1 receptor blockade in hypertensive patients with microinflammation. Circulation 110: 1103-1107, 2004. 
4. Chen FL, Yang ZH, Liu Y, et al: Berberine inhibits the expression of TNFalpha, MCP-1, and IL-6 in AcLDL-stimulated macrophages through PPARgamma pathway. Endocrine 33: 331-337, 2008.

5. Ni W, Kitamoto S, Ishibashi M, et al: Monocyte chemoattractant protein-1 is an essential inflammatory mediator in angiotensin II-induced progression of established atherosclerosis in hypercholesterolemic mice. Arterioscler Thromb Vasc Biol 24: 534-539, 2004

6. Schupp M, Janke J,Clasen R,UngerT and Kintscher U: Angiotensin type 1 receptor blockers induce peroxisome proliferator-activated receptor-gamma activity. Circulation 109: 2054-2057, 2004.

7. Lewis EJ, Hunsicker LG, Clarke WR, et al: Renoprotective effect of the angiotensin-receptor antagonist irbesartan in patients with nephropathy due to type 2 diabetes. N Engl J Med 345: 851-860, 2001.

8. Parving HH, Lehnert H, Brochner-Mortensen J, Gomis R, Andersen S and Arner P: The effect of irbesartan on the development of diabetic nephropathy in patients with type 2 diabetes. $\mathrm{N}$ Engl J Med 345: 870-878, 2001.

9. Lincoff AM, Wolski K, Nicholls SJ and Nissen SE: Pioglitazone and risk of cardiovascular events in patients with type 2 diabetes mellitus: a meta-analysis of randomized trials. JAMA 298: 1180$1188,2007$.
10. Janiak P, Bidouard JP, Cadrouvele C, et al: Long-term blockade of angiotensin AT1 receptors increases survival of obese Zucker rats. Eur J Pharmacol 534: 271-279, 2006.

11. Clasen R, Schupp M, Foryst-Ludwig A, et al: PPARgammaactivating angiotensin type-1 receptor blockers induce adiponectin. Hypertension 46: 137-143, 2005.

12. Toblli JE, Munoz MC, Cao G, Mella J, Pereyra L and Mastai R: ACE inhibition and AT1 receptor blockade prevent fatty liver and fibrosis in obese Zucker rats. Obesity (Silver Spring) 16: 770-776, 2008.

13. Adams LA and Feldstein AE: Nonalcoholic steatohepatitis: risk factors and diagnosis. Expert Rev Gastroenterol Hepatol 4: 623-635, 2010.

14. Munoz MC, Argentino DP, Dominici FP, Turyn D and Toblli JE: Irbesartan restores the in-vivo insulin signaling pathway leading to Akt activation in obese Zucker rats. J Hypertens 24: 1607-1617, 2006. 